\title{
Formation of Multicomponent Size-Sorted Assembly Patterns by Tunable Templated Dewetting
}

\author{
Dan Guo, Xu Zheng, * Xiaohe Wang, Huizeng Li, Kaixuan Li, Zheng Li, and Yanlin Song*
}

\begin{abstract}
Selective and deterministic assembly of particles is fundamentally significant for manufacturing functional devices. However, it is still a challenge to precisely and facilely manipulate particles of different sizes into different assembly patterns. Herein, a method is presented to achieve precise control over the formation of binary or ternary particle sizesorted assemblies. We investigate the assembly process of particles by capillary confinement and show that different sizesorted assemblies of multiple components can be realized by tuning the templated dewetting. By controlling the dewetting direction, receding contact angle, and pillar height of the template, assembly of dual-ring patterns, "comet" structures, and patterns with component separation are regulated. These structures can be further diversified by tuning the composition of the particles. This approach is general for particle assemblies of different sizes and materials, which will be significant for the fabrication of printed micro/nanohybrid devices.
\end{abstract}

The controllable assembly of desired architectures from micro- and nanoparticles is of great importance for materials science $^{[1]}$ and the manufacture of novel functional devices. ${ }^{[2]}$ In recent years, assembly of particles with different sizes, shapes, compositions, or a combination of these features has attracted widespread interest owing to the unique collective or synergistic effects of the assemblies ${ }^{[3]}$ such as applications in catalysis, ${ }^{[4]}$ chemical or biosensing, ${ }^{[5]}$ optoelectronics, ${ }^{[6]}$ and biomedicine. ${ }^{[7]}$ To date, extensive efforts have been made to explore the particle assembly mechanisms such as the entropic force and interparticle interaction. ${ }^{[8]}$ Entropydriven assembly and its combination with interparticle interactions have been widely studied in the formation of colloidal crystals or superlattices. ${ }^{[9]}$ Assembly of low-dimen-

[*] D. Guo, H. Li, K. Li, Z. Li, Prof. Y. Song

Key Laboratory of Green Printing, Institute of Chemistry

Chinese Academy of Sciences

Beijing 100190 (P. R. China)

and

Beijing Engineering Research Center of Nanomaterials for Green Printing Technology, Beijing National Laboratory for Molecular

Sciences

Beijing 100190 (P. R. China)

E-mail: ylsong@iccas.ac.cn

Dr. X. Zheng, X. Wang

State Key Laboratory of Nonlinear Mechanics

Institute of Mechanics, Chinese Academy of Sciences

Beijing 100190 (P. R. China)

E-mail: zhengxu@Inm.imech.ac.cn

(16) Supporting information and the ORCID identification number(s) for (D) the author(s) of this article can be found under:

https://doi.org/10.1002/anie.201810728. sional assemblies (e.g., particle clusters, chains, and mono- or bi-layers $)^{[10]}$ has been investigated by methods such as the use of heterogeneous surface modification to create "patchy" particles, ${ }^{[11]}$ electronic ${ }^{[12]}$ or magnetic ${ }^{[13]}$ field-induced assembly, DNA origami-templated assembly, ${ }^{[14]}$ and solvent-mediated phase separation. ${ }^{[15]}$ These strategies are based on particle modification or accurate control of the external field. Meanwhile, templated dewetting has been developed as an attractive paradigm for accurate spatial placement of the particle components over a large area, ${ }^{[16]}$ representing an effective technique that combines the high throughput of lithographic patterning and the high precision of component assembly. ${ }^{[17]}$ These rigid templates, however, have no selectivity for different particles and thus require multi-step experimental manipulation for multiple components. ${ }^{[18]}$ Recently, we have reported the programmed one-dimensional co-assembly strategy by soft liquid confinement. ${ }^{[19]}$ The capillary confinement of the meniscus interface serves as a soft template for the stepwise assembly of microparticles, facilitating the controllability of the assembled structures. In addition to the one-dimensional assembly, facilely manipulating multicomponent assembly of other complex structures or patterns needs to be addressed. Herein, an effective and facile strategy is demonstrated to achieve different size-sorted assembly patterns from binary and even ternary particles by tunable templated dewetting on a topographical substrate.

The assembly system is built by adding a mixed binary particle suspension onto a pillar template, ${ }^{[19]}$ covered by a superhydrophilic substrate (Figure $1 \mathrm{a}$; the experimental conditions and procedures are described in the Supporting Information). The gap $(G)$ between the cover and the pillar template is designed to be approximately $40 \mu \mathrm{m}$ by a microspacer. By a plasma-treated pillar template with the pillar height $(H)$ of $5 \mu \mathrm{m}$, the assembly process of binary particles with diameters of $1 \mu \mathrm{m}$ and $0.37 \mu \mathrm{m}$ (the volume fractions of the large and small particles are $0.3 \%$ and $0.2 \%$, respectively) is investigated (Figure $1 \mathrm{~b}$; the time is labeled in the bottom left of each picture). With the evaporation of the solvent, the receding suspension front gradually retreats and dewets the pillars. Gravity does not play an important role in the present microscale capillary dewetting process because the Bond number $\left(\mathrm{Bo}=\Delta \rho g G^{2} / \gamma_{\mathrm{LV}}\right)$, describing the contribution of gravity compared to surface tension, is estimated to be only $10^{-4}$. On a plasma-treated pillar template with a receding contact angle lower than $10^{\circ}$, the assembly process consists of particle size-sorting and recombining stages, finally forming a dual-ring structure as shown in Video S1. The size sorting of the binary particles is determined by the capillary confinement of the wedge-shaped meniscus on a hydrophilic substrate. ${ }^{[20]}$ As the receding three-phase contact line (TCL) 
a
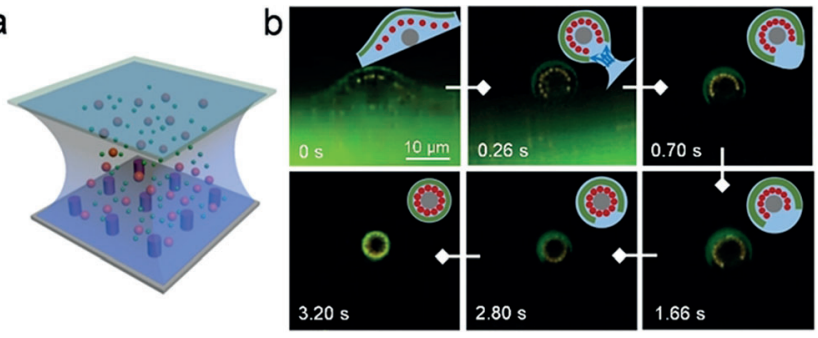

C
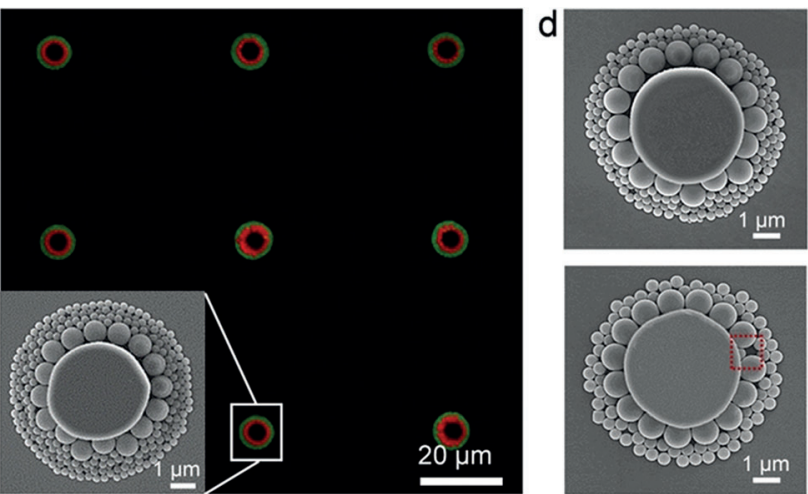

Figure 1. a) A suspension composed of particles of two sizes is sandwiched between a pillar template and a superhydrophilic cover. b) The binary particle assembly process during the suspension dewetting of the superhydrophilic pillar template, illustrated by the inset sketches. Blue arrows indicate the inner capillary flow. c) The laser scanning confocal microscope image and the inset SEM image show the dual-ring assembly patterns from particles with diameters of $1 \mu \mathrm{m}$ and $0.37 \mu \mathrm{m}$. d) Assemblies obtained by increasing $V f_{\mathrm{L} / \mathrm{S}}$ to 3 (top, decreasing the volume fraction of small particles from $0.2 \%$ to $0.1 \%$ ) or decreasing $D_{\mathrm{L} / \mathrm{S}}$ to 2.13 (bottom, increasing the diameter of the small particles from $0.37 \mu \mathrm{m}$ to $0.47 \mu \mathrm{m}$ ).

drags all the adjacent particles toward the pillar, the particles first form roughly ordered structures near the TCL; the smaller particles (green dots) align along the TCL near the end of the meniscus wedge, while the larger particles (red/ orange dots) loosely align relatively far from the end of the meniscus wedge owing to the geometry of the capillary confinement. When the receding suspension front contacts the pillar, the TCL will be curved. Then, the gas-liquid interface becomes discontinuous, and the TCL will warp around the pillar with the formation of a capillary neck. ${ }^{[21]}$ The hydrodynamic flow and the capillary force regulate the particles' positions and facilitate the compact size-sorted arrangement of particles along the radial direction of the pillar. After the rupture of the capillary neck, the TCL recovers the circular shape around the pillar driven by lowering the surface energy. Then, with the liquid shrinking owing to evaporation, large particles first assemble to surround the pillar, followed by the small particles gradually rearranging along the outer contour of the large particles. Figure 1c and the Supporting Information, Figure S4, show the resulting array of well-ordered dual-ring assemblies, in which the outer small-particle ring adjoins the inner largeparticle ring. This means that the capillary confinement effect serves to selectively arrange the binary particles into ordered structures. This method can produce uniform dual-ring structures in a large area of approximately $1.5 \times 1.5 \mathrm{~mm}^{2}$ containing $30 \times 30$ pillars. The dual-ring patterns can be varied by tuning the volume fraction ratios $\left(V \mathrm{f}_{\mathrm{L} / \mathrm{S}}\right)$ or the size ratios $\left(D_{\mathrm{L} / \mathrm{S}}\right)$ of large-to-small particles. Figure $1 \mathrm{~d}$ shows the different assemblies obtained by increasing $V \mathrm{f}_{\mathrm{L} / \mathrm{S}}$ (top image) or decreasing $D_{\mathrm{L} / \mathrm{S}}$ (bottom image). A larger $D_{\mathrm{L} / \mathrm{S}}$ facilitates the formation of a more compact dual-ring structure. If there are not enough large particles to surround the pillar, the vacancy will be filled by small particles, as shown in the red frame in Figure $1 d$.

The capillary confined space between the meniscus gasliquid interface and the template/pillar surface around the pillar is critical in the assembly of the dual-ring pattern. When the receding gas-liquid interface approaches and comes into contact with the pillar, the profile of the interface will become discontinuous (Figure $2 \mathrm{a}$; the surfaces of both the cover and template are superhydrophilic). Assuming that the receding contact angle on the template is approximately $0^{\circ}$, the curvature radius of the meniscus is considered approximately equal to the pillar height $H{ }^{[21]}$ This indicates that the capillary confined space for the assembly of the dual-ring structures is

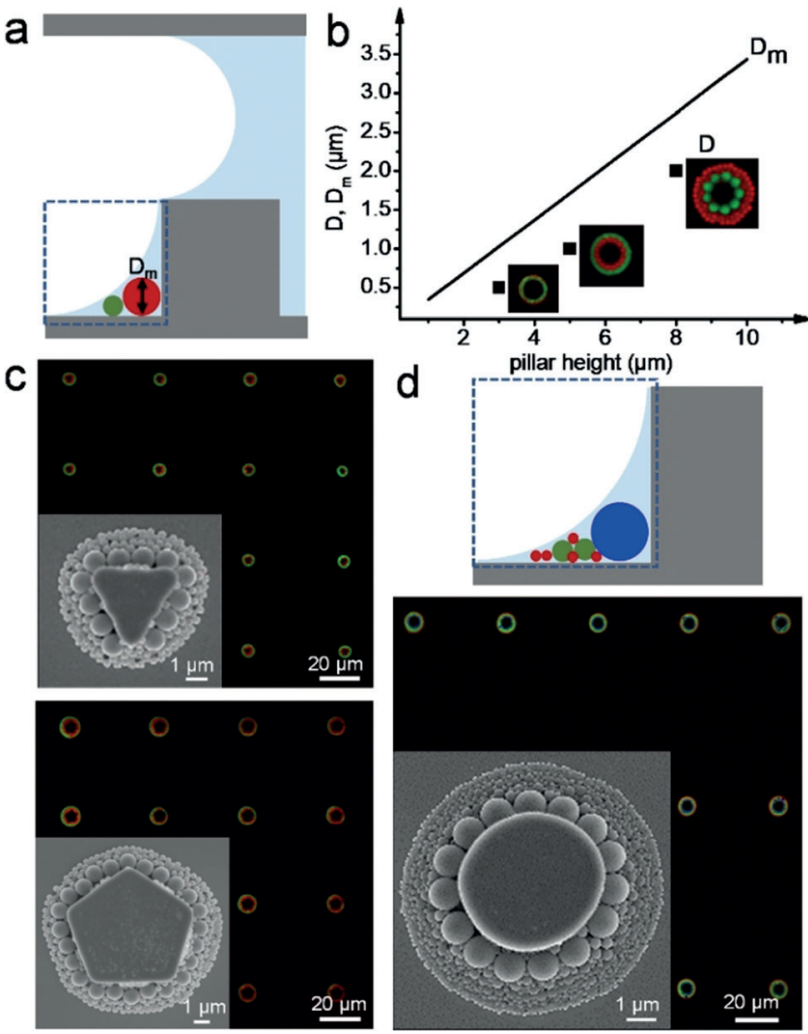

Figure 2. a) Schematic of two-sized particles in the capillary confinement upon the air-liquid interface becoming discontinuous. b) Plot of $D_{\mathrm{m}}$ (black line) and $D$ (squares) versus $H$, illustrating that both $D_{\mathrm{m}}$ and $D$ show a positive correlation with $H$ and that $D$ is smaller than $D_{\mathrm{m}}$. c) The confocal micrographs and SEM images show the dual-ring assemblies in triangular and pentagonal patterns. d) Schematic of ternary (three-sized) particles in the capillary confinement near the pillar, confocal micrograph of the ternary-ring assembly array and the inset SEM image, showing that the largest particles of $1 \mu \mathrm{m}$ constitute the innermost ring, the smallest particles of $0.18 \mu \mathrm{m}$ constitute the outermost ring, and the medium-sized particles of $0.37 \mu \mathrm{m}$ mixed with the smallest particles constitute the middle ring. 
actually determined by $H$. The theoretical maximum diameter of large particles that can be filled into the capillary confined space $\left(D_{\mathrm{m}}\right)$ can be expressed by $D_{\mathrm{m}} / H=2(\sqrt{2}-1) /(\sqrt{2}+1) \approx 0.34$, which is shown by the solid line in Figure $2 \mathrm{~b}$. In the experiments, the actual diameters of the large particles $(D$, marked by three dots in Figure $2 \mathrm{~b}$ ) show a similar trend as the theoretical line. The positive correlation between $D_{\mathrm{m}}(D)$ and $H$ indicates that the template with the larger pillar height allows the assembly of the particles with a larger size. $D$ is smaller than $D_{\mathrm{m}}$ mainly because the exceeded additional space is needed to provide room for the regulation of particle assembly in the recombining stage. This suggests that $D / H$ should be smaller than 0.34 to achieve assembly of the dual-ring structures. Moreover, the theoretical maximum diameter of the small particles $\left(d_{\mathrm{m}}\right)$ can also be derived as $1 / \sqrt{ }\left(d_{m}\right)=1 / \sqrt{ }\left(D_{m}\right)+1 / \sqrt{ } H$. When the diameter of large particles $D$ is much smaller than $D_{\mathrm{m}}$, there will be more than one layer of large particles, and small particles are prone to being trapped in the interstices of large particles (Figure S5). With the template having different shapes of the pillar cross-sections, different patterns with large particles arranging along the convex edges can be achieved (Figure 2c), showing the potential of our method to construct more complex structures. Furthermore, ternary particle assembly is investigated. It is found that the largest particles constitute the innermost ring and that the smallest particles constitute the outermost ring, while the mediumsized particles in the middle region are mixed with the smallest particles (Figure 2d and Figure S6). This is because the smallest particles can easily be restricted in the interstices among the larger particles. ${ }^{[22]}$

By tuning the capillary confinement effect and the suspension dewetting direction, another size-sorted assembly along the dewetting direction can be realized, and a series of comet structures are achieved. Figure 3 a illustrates the variation of the capillary confined space as the suspension dewets the pillar. According to our model (Supplementary discussion 1), this capillary confined space increases with both $H$ and the gap $G$. Therefore, we can tune the capillary confinement effect by varying the geometric factors $H$ and $G$. When $G<2 H$, the decrease of the capillary space will lead to incomplete assembly as the space in front of the pillar is not large enough for large particle assembly [Eq. (S3) in supplementary discussion 1]. On the other hand, when $G \gg 2 H$, the capillary confined space is too large to enable the size sorting of particles owing to the weak confinement effect. Decreasing $G$ can significantly increase the liquid dewetting speed [Eq. (S7)]. However, a dewetting speed that is too fast is not desired as it would drag more particles away from the vicinity of the pillar. As a result, we set $G \approx 40 \mu \mathrm{m}$ to optimize the experiment. Furthermore, the dewetting direction of the suspension can be controlled by tuning the tilted angle of the cover (Supplementary discussion 2). By simply establishing a small tilted angle, which is in the range of 0.001-0.005 radians, we can achieve a controllable dewetting speed (approximately $1 \mathrm{~cm} \mathrm{~s}^{-1}$ to $5 \mathrm{~cm} \mathrm{~s}^{-1}$ ) and direction due to the capillary pressure difference of the meniscus at the two ends. Along the dewetting direction, we name the capillary confined space before the TCL receding across the pillar the "front" region,

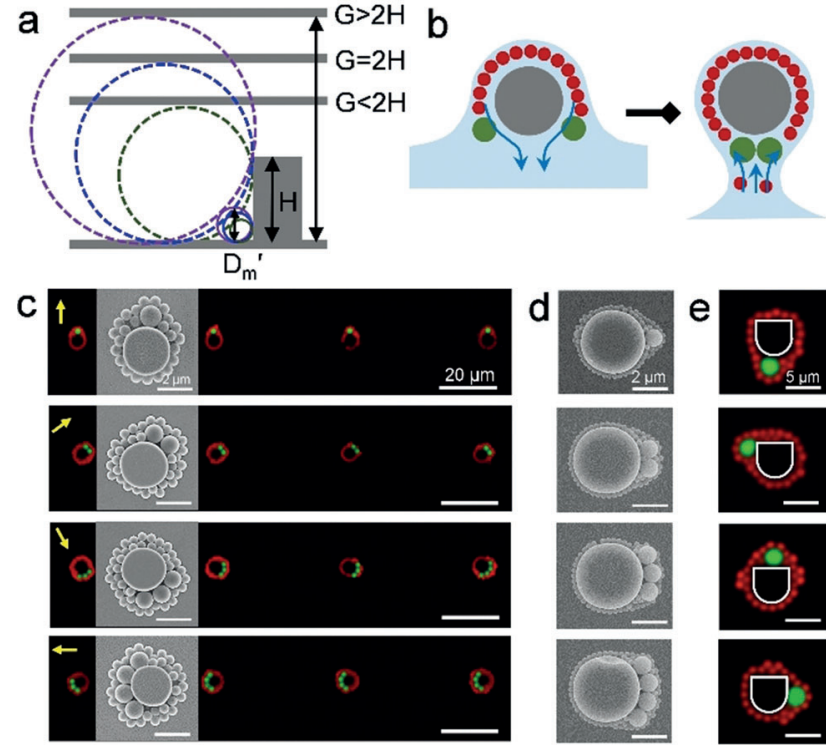

Figure 3. The change of capillary confined space and the formation of "comet" structures. a) Side-view of binary particles and capillary meniscus near the pillar upon contact of the gas-liquid interface with the pillar. The maximum containable particle diameter $D_{\mathrm{m}}{ }^{\prime}$ in the capillary confinement is influenced by $H$ and $G$. b) Top-view of the comet structure formation: large particles are pushed to the rear region by the interface, and small particles are convectively confined to assemble surrounding the pillar and large particles. c) The formed comet structures consist of large particles at tunable locations. Yellow arrows indicate the dewetting direction. d) Particles of $1 \mu \mathrm{m}$ and $0.29 \mu \mathrm{m}$ are assembled in comet structures when the pillar height is reduced to $2.5 \mu \mathrm{m}$. e) Different assembly patterns with precisely controlled location of the large particle on the noncentrosymmetric pillar template.

and that behind the pillar the "rear" region. When the ratio of the large particle diameter to the pillar height $D / H$ exceeds 0.34 , the large particles near the pillar cannot stay in the front region but will be pushed to the rear region by the interface. The small particles follow the loop-assembly to surround the pillar and the corresponding large particles (Figure $3 \mathrm{~b}$ ). For instance, $1 \mu \mathrm{m}$ and $2 \mu \mathrm{m}$ particles with the volume fractions of $0.3 \%$ and $0.12 \%$, respectively, can be assembled into comet structures by the template with $H=5 \mu \mathrm{m}$. The $2 \mu \mathrm{m}$ particles are directionally located in the rear region, and the $1 \mu \mathrm{m}$ particles assemble surrounding the pillar and $2 \mu \mathrm{m}$ particles. Furthermore, with the gradual increase in the volume fraction of large particles from approximately $0.12 \%$ to $0.48 \%$, the number of large particles in the assembled comet structures can be correspondingly increased from one to four (Figure $3 \mathrm{c}$, Experimental procedures in the Supporting Information, and Videos S2-S5). The fluctuation in the patterns of the small particles is sometimes noticeable, which is mainly due to the local non-uniform density of the small particles, influenced by both Brownian motion and capillary flow. Similarly, particles with diameters of $1 \mu \mathrm{m}$ and $0.29 \mu \mathrm{m}$ can also be assembled into the comet structures when $H$ is reduced to $2.5 \mu \mathrm{m}$ (Figure $3 \mathrm{~d}$ ). Thus, we can diversify the comet structures by controlling the value of $D / H$ to exceed 0.34 and varying the sizes of the binary particles (Figure S7). Multicomponent 
particle sorting along a controlled direction is also achieved by the pillar template with a noncentrosymmetric crosssection (Figure 3e). Different assembly patterns can be achieved with the same template by simply tuning the dewetting direction, which can be applied to diversify the assembly patterns and fabricate special structures.

Differently, patterns with component separation can be achieved by the template with a receding contact angle larger than $45^{\circ}$. The confined space in front of the pillar is decreased with the receding contact angle for two reasons. First, the TCL on the relatively hydrophobic template is located closer to the pillar than the TCL on the hydrophilic template, leaving a diminished capillary confined space (Figure S8). Second, the TCL recedes more easily on the hydrophobic template than that on the hydrophilic template. ${ }^{[23]}$ Figure $4 \mathrm{a}, \mathrm{b}$
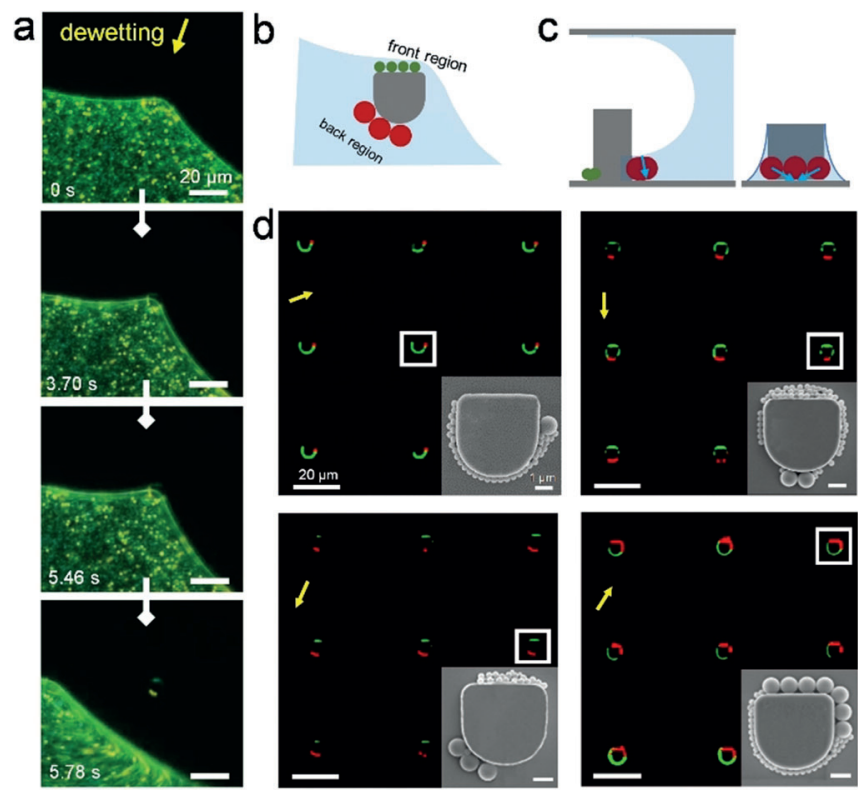

Figure 4. a) The images show the separation process for particles of two sizes on the pillar template with a receding contact angle larger than $45^{\circ}$. b,c) The top- and side-views of the confined assembly of the small and large particles at the front and rear region of the pillar, respectively. The blue arrows indicate the capillary force on the confined particles. d) The confocal micrographs and the inset magnified SEM images show different patterns with the separation of $1 \mu \mathrm{m}$ and $0.37 \mu \mathrm{m}$ particles. The yellow arrows indicate the dewetting direction.

(the time is labeled in the bottom left of each snapshot) shows the component separating process; in the beginning when the TCL approaches the pillar, only small particles (the aligned green dots near the TCL) can be confined to assemble at the front region near the receding TCL, as the space is not large enough to accommodate large particles (red/orange dots). Thus, the large particles aggregate only at the rear region (Video S6). When the TCL lastly recedes across the pillar, the large particles in the rear region of the pillar are assembled under the capillary force (Figure $4 \mathrm{c}$ ). The dewetting direction across the pillar determines the placement of the large particles. Different from the comet structure in which small particles surround both the pillar and large particles, the small particles cannot surround the large particles, mainly owing to the reduced capillary confined space on the relatively hydrophobic template, as explained above. Additionally, the smaller particles will be more easily carried away by the fast dewetting flows instead of staying in the rear region owing to their low inertia. Thus, large and small particles are thoroughly separated by the pillar along the dewetting direction. Employing a pillar template with a noncentrosymmetric cross-section, the oriented patterns can be precisely controlled by simply tuning the suspension dewetting direction through establishing a tilted angle (Figure $4 \mathrm{~d}$ and Figure S9). In contrast to the previously reported separation methods for differently sized particles, which can only be realized on a sufficiently hydrophilic substrate, ${ }^{[20]}$ we achieve size separation of particles on a hydrophobic substrate and can also precisely control the assembly patterns. This facile strategy shows generality for the particle assembly of different materials (Figure S10), which achieves good and consistent results using particles with a range of diameters from approximately $0.1 \mu \mathrm{m}$ to $2 \mu \mathrm{m}$. Based on this, developing hybrid nanophotonics can be expected by the dual-ring assembly of particles with different materials, such as enhanced luminescence by the combination of plasmonic nanostructures and phosphor, ${ }^{[24]}$ and enhanced harmonic generation by the interplay of plasmonic particles and dielectric high refractive-index particles with non-linear properties. ${ }^{[25]}$

In conclusion, we demonstrate a facile strategy to achieve precise control of multicomponent particle assembly for different patterns. By controlling the receding contact angle and the pillar height of the template, the capillary confined effect can be tuned to control different size-sorted assemblies of multiple components near the receding TCL. Accordingly, particle assembly of dual-ring structures, comet structures, and patterns with component separation are achieved. In each type of assembly, the patterns are diversified by tuning the composition of particles. Furthermore, multicomponent sizesorted assembly along a desired direction can be precisely controlled by regulating the suspension dewetting direction. This effective and facile approach can be generalized for different particle assemblies with various compositions to achieve desired patterns, which will have promising applications in the fabrication of functional devices based on material assembly.

\section{Acknowledgements}

The authors thank the financial support from the National Key R\&D Program of China (Grant Nos. 2018YFA020850, 2016YFB0401603, 2016YFC1100502 and 2016YFB0401100), the National Nature Science Foundation (Grant Nos. 51773206, 51473173, and 11572335), the Strategic Priority Research Program of the Chinese Academy of Sciences (Grant No. XDA09020000, XDB22040403), and the CAS Key Research Program of Frontier Sciences (QYZDB-SSWJSC036). 


\section{Conflict of interest}

The authors declare no conflict of interest.

Keywords: capillary assembly · confinement · dewetting · patterns $\cdot$ size-sorting

[1] a) F. Li, D. P. Josephson, A. Stein, Angew. Chem. Int. Ed. 2011 , 50, 360-388; Angew. Chem. 2011, 123, 378-409; b) M. H. Lash J. C. Jordan, L. C. Blevins, M. V. Fedorchak, S. R. Little, J. J. McCarthy, Angew. Chem. Int. Ed. 2015, 54, 5854-5858; Angew. Chem. 2015, 127, 5952-5956; c) M. A. Boles, M. Engel, D. V. Talapin, Chem. Rev. 2016, 116, 11220-11289; d) W. Ma, L. Xu, A. de Moura, X. Wu, H. Kuang, C. Xu, N. A. Kotov, Chem. Rev. 2017, 117, $8041-8093$.

[2] a) C. R. Kagan, E. Lifshitz, E. H. Sargent, D. V. Talapin, Science 2016, 353, aac5523; b) J.-H. Choi, H. Wang, S. J. Oh, T. Paik, P. Sung, J. Sung, X. Ye, T. Zhao, B. T. Diroll, C. B. Murray, Science 2016, 352, 205-208; c) S. Ortega, M. Ibáñez, Y. Liu, Y. Zhang, M. V. Kovalenko, D. Cadavid, A. Cabot, Chem. Soc. Rev. 2017, $46,3510-3528$.

[3] a) M.-P. Pileni, Acc. Chem. Res. 2017, 50,1946-1955; b) C. Yan, T. Wang, Chem. Soc. Rev. 2017, 46, 1483; c) D. Guo, Y. Song, Chem. Eur. J. 2018, 24, 1-14.

[4] Y. Kang, X. Ye, J. Chen, Y. Cai, R. E. Diaz, R. R. Adzic, E. A. Stach, C. B. Murray, J. Am. Chem. Soc. 2013, 135, 42-45.

[5] a) M. P. Cecchini, V. A. Turek, J. Paget, A. A. Kornyshev, J. B. Edel, Nat. Mater. 2013, 12,165-171; b) X. Wu, L. Xu, W. Ma, L. Liu, H. Kuang, N. A. Kotov, C. Xu, Adv. Mater. 2016, 28, $5907-$ 5915.

[6] a) J. J. Urban, D. V. Talapin, E. V. Shevchenko, C. R. Kagan, C. B. Murray, Nat. Mater. 2007, 6, 115-121; b) Y. G. Zhang, F. Lu, K. G. Yager, D. van der Lelie, O. Gang, Nat. Nanotechnol. 2013, $8,865-872$.

[7] a) T. Sun, Y. S. Zhang, B. Pang, D. C. Hyun, M. Yang, Y. Xia, Angew. Chem. Int. Ed. 2014, 53, 12320-12364; Angew. Chem. 2014, 126, 12520-12568; b) Y. S. Zhang, C. Zhu, Y. Xia, $A d v$. Mater. 2017, 29, 1701115.

[8] a) D. Frenkel, Nat. Mater. 2015, 14, 9-12; b) Y. Min, M. Akbulut, K. Kristiansen, Y. Golan, J. Israelachvili, Nat. Mater. 2008, 7, 527-538.

[9] a) P.-P. Wang, Q. Qiao, Y. Zhu, M. Ouyang, J. Am. Chem. Soc. 2018, 140, 9095-9098; b) Y. Wang, Y. F. Wang, X. L. Zheng, E. Ducrot, J. S. Yodh, M. Weck, D. J. Pine, Nat. Commun. 2015, 6 , 7253; c) S. Y. Park, A. K. R. Lytton-Jean, B. Lee, S. Weigand, G. C. Schatz, C. A. Mirkin, Nature 2008, 451, 553-556.

[10] L. Cademartiri, K. J. Bishop, Nat. Mater. 2015, 14, 2 -9.

[11] a) L. Xu, H. Kuang, C. Xu, W. Ma, L. Wang, N. A. Kotov, J. Am. Chem. Soc. 2012, 134, 1699-1709; b) M. R. Jones, N. C. Seeman, C. A. Mirkin, Science 2015, 347, 1260901; c) X. Zheng, M. Liu,
M. He, D. J. Pine, M. Weck, Angew. Chem. Int. Ed. 2017, 56 , 5507-5511; Angew. Chem. 2017, 129, 5599-5603; d) Z. Gong, T. Hueckel, G.-R. Yi, S. Sacanna, Nature 2017, 550, 234-238.

[12] K. L. Heatley, F. Ma, N. Wu, Soft Matter 2017, 13, 436-444.

[13] R. M. Erb, H. S. Son, B. Samanta, V. M. Rotello, B. B. Yellen, Nature 2009, 457, 999-1002.

[14] Y. Tian, T. Wang, W. Liu, H. L. Xin, H. Li, Y. Ke, W. M. Shih, O. Gang, Nat. Nanotechnol. 2015, 10, 637-644.

[15] A. H. Gröschel, A. Walther, T. I. Löebling, F. H. Schacher, H. Schmalz, A. H. E. Müeller, Nature 2013, 503, 247-251.

[16] a) R. A. Hughes, E. Menumerov, S. Neretina, Nanotechnology 2017, 28, 282002; b) V. Flauraud, M. Mastrangeli, G. D. Bernasconi, J. Butet, D. T. Alexander, E. Shahrabi, O. J. Martin, J. Brugger, Nat. Nanotechnol. 2017, 12, 73-80; c) K. Xu, L. Qin, J. R. Heath, Nat. Nanotechnol. 2009, 4, 368-372.

[17] a) A. M. Hung, C. M. Micheel, L. D. Bozano, L. W. Osterbur, G. M. Wallraff, J. N. Cha, Nat. Nanotechnol. 2010, 5, 121-126; b) L. Jiang, X. Chen, N. Lu, L. Chi, Acc. Chem. Res. 2014, 47, 3009-3017; c) M. Asbahi, S. Mehraeen, K. T. Lim, F. Wang, J. Cao, M. C. Tan, J. K. Yang, Nano Lett. 2014, 14, 2642-2646.

[18] a) Q.-Y. Lin, J. A. Mason, Z. Li, W. Zhou, M. N. O’Brien, K. A. Brown, M. R. Jones, S. Butun, B. Lee, V. P. Dravid, K. Aydin, C. A. Mirkin, Science 2018, 359, 669-672; b) S. Ni, J. Leemann, I. Buttinoni, L. Isa, H. Wolf, Sci. Adv. 2016, 2, e1501779; c) Y. D. Yin, Y. Lu, Y. N. Xia, J. Am. Chem. Soc. 2001, 123, 771-772.

[19] a) D. Guo, C. Li, Y. Wang, Y. Li, Y. Song, Angew. Chem. Int. Ed. 2017, 56, 15348-15352; Angew. Chem. 2017, 129, 15550-15554; b) D. Guo, Y. Li, X. Zheng, F. Li, S. Chen, M. Li, Q. Yang, H. Li, Y. Song, J. Am. Chem. Soc. 2018, 140, 18-21.

[20] a) T.-S. Wong, T.-H. Chen, X. Shen, C.-M. Ho, Anal. Chem. 2011, 83, 1871-1873; b) W. Han, M. Byun, Z. Lin, J. Mater. Chem. 2011, 21, 16968-16972.

[21] C. Gögelein, M. Brinkmann, M. Schröter, S. Herminghaus, Langmuir 2010, 26, 17184-17189.

[22] M. H. Kim, S. H. Im, O. O. Park, Adv. Mater. 2005, 17, $2501-$ 2505.

[23] M. Kuang, J. Wang, B. Bao, F. Li, L. Wang, L. Jiang, Y. Song, $A d v$. Opt. Mater. 2014, 2, 34-38.

[24] a) M. Saboktakin, X. Ye, U. K. Chettiar, N. Engheta, C. B. Murray, C. R. Kagan, ACS Nano 2013, 7, 7186-7192; b) N. J. Greybush, M. Saboktakin, X. Ye, C. D. Giovampaola, S. J. Oh, N. E. Berry, N. Engheta, C. B. Murray, C. R. Kagan, ACS Nano 2014, $8,9482-9491$.

[25] a) B. Metzger, M. Hentschel, T. Schumacher, M. Lippitz, X. Ye, C. B. Murray, B. Knabe, K. Buse, H. Giessen, Nano Lett. 2014, 14, 2867-2872; b) F. Timpu, N. R. Hendricks, M. Petrov, S. Ni, C. Renaut, H. Wolf, L. Isa, Y. Kivshar, R. Grange, Nano Lett. 2017, $17,5381-5388$.

Manuscript received: September 18, 2018

Revised manuscript received: October 11, 2018

Version of record online: 


\section{Communications}

\section{Nanotechnology}

D. Guo, X. Zheng,* X. Wang, H. Li, K. Li,

Z. Li, Y. Song*

IIII-IIII

Formation of Multicomponent SizeSorted Assembly Patterns by Tunable Templated Dewetting
Guided assembly: An efficient method is presented to achieve precise control over the particle size-sorted assembly of different patterns from binary or ternary mixtures by controlling the templated dewetting behavior. The method is used to generate dual-ring patterns, "comet" structures, and separated component patterns. 